\title{
Control Space Reduction and Real-Time Accurate Modeling of Continuum Manipulators Using Ritz and Ritz-Galerkin Method
}

\author{
S.M.Hadi Sadati ${ }^{1}$, S. Elnaz Naghibi ${ }^{2}$, Ian D. Walker ${ }^{3}$, Kaspar Althoefer ${ }^{2}$, Thrishantha Nanayakkara $^{4}$
}

\begin{abstract}
To address the challenges with real-time accurate modeling of multi-segment continuum manipulators in presence of significant external and body loads, we introduce a novel series solution for variable-curvature Cosserat rod static and Lagrangian dynamic method. By combining a modified Lagrange polynomial series solution, based on experimental observations, with Ritz and Ritz-Galerkin methods, the infinite modeling state space of a continuum manipulator is minimized to geometrical position of a handful of physical points (in our case two). As a result, a unified easy to implement vector formalism is proposed for nonlinear impedance and configuration control. We showed that by considering the mechanical effects of axial highly elastic deformation, the model accuracy is increased by up to $6 \%$. The proposed model predicts experimental results with 6-8\% (4-6 [mm]) mean error for the Ritz-Galerkin method in static cases and $16-20 \%(12-14[\mathrm{~mm}])$ mean error for the Ritz method in dynamic cases, in planar and general 3D motions. Comparing to five different models in the literature, our approximate solution showed to be more accurate with the smallest possible number of modeling states and suitable for real-time modeling, observation and control applications.
\end{abstract}

Index Terms-Flexible robots, Soft Material Robotics, Dynamics, Motion Control, Force Control.

\section{INTRODUCTION}

$\mathbf{T}$ AKING inspiration from biological examples such as the octopus arms, chameleon tongues and elephant trunks, researchers are looking into the possibility of replicating similar maneuverability and grasping characteristics by harnessing the corresponding hyper-redundancy demonstrated in nature [1]. The class of continuum robots promises considerable performance improvements in different areas which currently

Manuscript received: March, 21, 2017; Revised July, 4, 2017; Accepted July, 30, 2017.

This paper was recommended for publication by Editor Paolo Rocco upon evaluation of the Associate Editor and Reviewers' comments. *This work is supported in part by the U.K. Engineering and Physical Sciences Research Council (EPSRC) under Grant EP/N03211X/2, and European Union H2020 project FourByThree code 637095

${ }^{1}$ S.M.Hadi Sadati is with The Centre for Robotics Research (CoRe), Department of Informatics, King's College London, London WC2R 2LS, United Kingdom. (Corresponding author e-mail: seyedmohammadhadi.sadati@kcl.ac.uk)

${ }^{2}$ S. E. Naghibi and K. Althoefer are with the School of Engineering and Materials Science, Queen Mary University of London, Mile End Road, London E1 4NS, United Kingdom. (/s.e.naghibi, k.althoefer/ (domul.ac.uk)

${ }^{3}$ Ian D. Walker is with the Department of Electrical and Computer Engineering, Clemson Univer-sity, Clemson, USA. (iwalkereg.clemson.edu)

${ }^{4}$ Thrishantha Nanayakkara is with the Dyson School of Design Engineering, Imperial College London, 10 Princes Gardens, London, SW7 1NA. (t.nanayakkara@imperial.ac.uk)

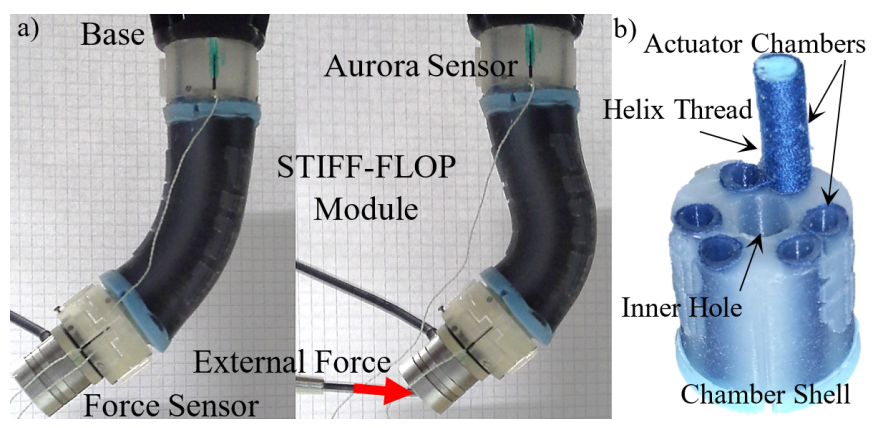

Fig. 1. A STIFF-FLOP continuum actuator module in experiments with significant external loads (a), module structure details (b).

witness the presence of traditional robots, such as surgical applications, underwater manipulation and inspection, etc. [2], [3], [4]. Real-time accurate modeling, observation and controller design suitable for this class of manipulators are shown to be complex and challenging tasks due to their high dexterity and deformability [5].

A Continuum manipulator model is described using a general kinematic map for the strain-configuration relation and a robot-specific mechanical map for the loads-strain relation. The Constant curvature (CC) model, which is the most common assumptions in continuum robotic research, simplifies the kinematics of a continuum manipulator by expressing the backbone deformation as a planar CC profile [5]. Although being commonly used as a simplifying assumption, the CC assumption suffers from local singularities and low accuracy in the presence of significant body and external loads. Variable curvature (VC) [6], [7] and identification based seriessolutions [8] provide more accurate singularity free kinematic maps. The dynamic models introduced in the literature to provide a better modeling accuracy can be categorized into six groups, a comparative study of which in comparison with experiments is presented in [9]. 1) Lumped system Lagrange dynamics with discrete $\mathrm{VC}$ kinematics, similar to a series rigid link mechanism, where a series of finite or infinite number of rigid disks are interconnected using compliant joints [10]. 2) Euler-Bernoulli beam mechanics with CC [11] or discrete VC kinematics [12]. 3) Continuum form of Lagrange dynamics [8], [13] or the Principle of Virtual Work (PVW) [14], [15] using $\mathrm{CC}$, continuous $\mathrm{VC}$ or series-solutions as kinematic maps, where the kinematic map parameters are the dynamic model states. 4) Cosserat rod model [6], [16] and beam theory method, as a simplified version of that [9], which result in a 
boundary value problem (BVP) to be solved using numerical optimization methods [6], [17]. 5) approximate identification based series-solutions where coefficients of a simple [18] or complex [19] series are identified using experimental results to construct a hardware-specific model. 6) Numerical finite element methods using Euler-Bernoulli [20] or Galerkin weakform series-solution [21] mechanics and VC kinematics.

The identification based models are more accurate and computationally efficient; however, their validity is limited to their experiment conditions, external loads, input values and training data set [19], [8]. Besides, they do not account for the structural characteristics necessary for dealing with unknown conditions and design optimization [9]. On the other hand, the lumped system and Cosserat rod models provide a general solution, but not suitable for control design due to high computational cost and large number of modeling states. Use of series-solution based models for identification of kinematic map by Godage [8] have shown to be realtime but with a complex hard to interpret shape function and limitations related to identification based methods. Seriessolution based Galerkin method for static modeling of complex deformation in a discretized finite element domain by Tunay [21] is accurate and comprehensive, but with a complex shape function and limitations related to finite element and Cosserat rod method.

Accuracy of these methods is compared based on their forward control performance [9] and a feedback term, such as PID, should be considered in the controller design to deal with real working disturbances. The large modeling state space results in hard impedance observation and control which are essential for safe surgical, inspection and human-robot interaction applications.

In our previous research [14], [15], we introduced a new geometry deformation based approach for the inverse static model of braided continuum manipulators. We utilized the experimental observation of the deformed cross-section to model the deformation energy of the continuum media and to show the importance of considering the cross-section highly elastic deformation, using the PVW and the CC kinematics in [14] and Cosserat rod method with VC kinematics in [9]. For the first time, our model provides an accurate semianalytical solution for the manipulator cross-section deformation to increase the modeling accuracy, especially in the presence of regional coaxial homogeneous stiffness variation in the cross-section. There, different modeling assumptions are investigated, showing the advantageous of an exact analytical solution for design optimization based on sensitivity analysis of structural parameters. However, a controller was hard to design due to the infinite number of the modeling states.

In this paper, 1) we introduce a model with finite number of states while preserving the modeling accuracy and generality based on which the design of a nonlinear impedance and configuration feedback controller is possible; 2 ) the solution is extended to the dynamic case based on a vector formalism for PVW, in general 3D motions of a multi-segment manipulator; 3) the modeling accuracy is increased by considering the mechanical effects of the manipulator axial highly elastic deformation as well as in the planar direction. We introduce a novel, accurate yet real-time approximate series-solution for the Cosserat beam method in the static case and a PVW based model in the dynamic case for the general 3D motion of a multi-segment continuum manipulator in the presence of significant external and body loads. Based on the experimental observations, a modified Lagrange polynomial, as a differentiable polynomial of finite order, is chosen to drive a weak-form series-solution for the mechanics of a continuum manipulator using continues Ritz and Ritz-Galerkin methods. To the best of our knowledge and compared to the most recent similar research in the field [17], this is the first time that the infinite modeling state space of such problem minimizes to the geometrical positions of two points at the tip and in the middle of the manipulator. As a result, different dynamic impedance and configuration control scenarios are formulated using traditional nonlinear control theories in a unified and easy to implement vector formalism. We neglect the close loop terms in the first part to highlight the performance and accuracy of our method. A PID term is incorporated it in inverse control model later. Modeling results are verified against experimental results in the static and dynamic cases. The proposed model is the most accurate mechanics of materials based model with the smallest possible number of modeling states compared to the other well-known approaches in the literature [9] and can be used for precise static and dynamic modeling, observation and control in real-time applications.

In the following sections, first, the modeling framework is discussed consisting of the VC kinematics, the Cosserat and beam theory static model and PVW dynamic model. The Ritz and Ritz-Galerkin series solutions are detailed in section III for static and dynamic models followed by a vector form derivation for the nonlinear control and observation problems in section IV. Experiments and discussion are described in section $\mathrm{V}$ and conclusions are presented in section VI.

\section{MODELING FRAMEWORK}

Slenderness and compliance of most continuum manipulators make the external and body forces important in the modeling of their behavior. We use VC kinematics to relate a series-solution geometry in 3D space $\left(\in R^{3}\right)$ to the strain rates that are found from the Cosserat rod static model and to calculate the PVW terms for the dynamic model. To incorporate the mechanical effect of the manipulator highly elastic deformation, we assume a symmetric uni-axial deformation for the constant volume manipulator to update the module shell deformed cross-section radius $(r)$, area $(a)$, moment of inertia $(J)$, elasticity $(E)$ and shear $(G)$ modulus as $r_{\mathrm{d}}=r / \sqrt{\lambda_{\hat{d}_{1}}}$, $a_{\mathrm{d}}=a / \lambda_{\hat{d}_{1}}, J_{\mathrm{d}}=J / \lambda_{\hat{d}_{1}}^{2}$ and $E\left|G_{\mathrm{d}}=\lambda_{\hat{d}_{1}} E\right| G$, where $\lambda_{\hat{d}_{1}}$ is the local axial strain rate and the deformed state is shown by subscript ( d) [22], [9]. The pneumatic chamber crosssection is assumed to be constant due to dense braiding [14], [23]. Dependent variables are functions of space and time $(s, t)$ unless specified.

\section{A. Variable Curvature Kinematics}

The VC kinematics use curvilinear frames, $\left[\hat{d}_{2}, \hat{d}_{1}, \hat{d}_{3}\right]$ for cross-section physical frame or $[\hat{n}, \hat{t}, \hat{b}]$ for Frenet - Serret 


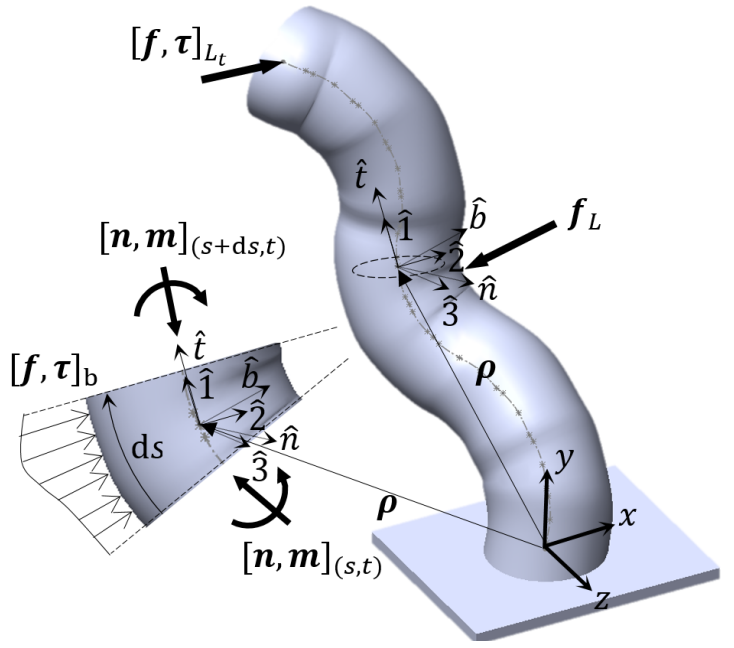

Fig. 2. Variable curvature kinematics and the Cosserat model free body diagram for one element. [ $\left[\hat{d}_{2}, \hat{d}_{1}, \hat{d}_{3}\right]$ is the physical, $[\hat{n}, \hat{t}, \hat{b}]$ is the Frenet - Serret and $[x, y, z]$ is the inertial frame. Subscript $L, L_{\mathrm{t}}$ and $b$ are for the tip load, general external load and body load respectively.

frame (Fig. 2), to represent the continuum manipulator backbone as a series of infinitesimal CC curves [7], [16], [6]. VC Kinematics present the relation between the physical curvilinear coordinates $\left(\left[\hat{d}_{2}, \hat{d}_{1}, \hat{d}_{3}\right]\right)$ and the curve spatial configuration $(\rho)$ expressed in inertial Cartesian coordinates $([\hat{i}, \hat{j}, \hat{k}])$. The physical frame $\left[\hat{d}_{2}, \hat{d}_{1}, \hat{d}_{3}\right]$, where $\hat{d}_{1}$ is tangent to the backbone and $\hat{d}_{2}$ is alongside the first air pressure chamber, is used in the forward VC kinematics. A $3 \times 3$ rotation matrix $(R)$ relates the local linear strain rates in the physical curvilinear coordinates $(v)$ to the spatial deformation rates of the manipulator geometry in the inertial Cartesian coordinates $\left(\rho_{, s}\right)$ as

$$
\rho_{, s}=R v+\hat{d}_{1}=R\left(v+\left[\begin{array}{ll}
0,1, & 0
\end{array}\right]^{\mathrm{T}}\right),
$$

where subscript $\left({ }_{x}\right)$ means partial differentiation w.r.t. variable $x$. The spatial variation of $R$ is found based on the local rotational strain rates $(u)$

$$
R_{, s}=[u]_{\times} R,
$$

and the angular velocity of each element is $[\omega]_{\times}=R_{, t} R$, where []$_{\times}$is an operator to create a skew symmetric matrix. The curvilinear and Cartesian coordinates are aligned at the manipulator base $(s=0)$ where $\rho_{0}=0, R_{0}=I$ and $I$ is a $3 \times 3$ unit matrix.

For the inverse $\mathrm{VC}$ kinematics, where the manipulator geometry is known as $\rho^{*}$, we use Frenet - Serret curvilinear frames with tangent $(\hat{t})$, normal $(\hat{n})$ and binormal $(\hat{b})$ unit vectors. Here, $\hat{b}$ expresses the direction of the local bending vector. The rotation matrix to relate Frenet - Serret to Cartesian coordinates is $R^{*}=[\hat{n}, \hat{t}, \hat{b}]$, where $\hat{t}=\rho_{, s}^{*} /\left|\rho_{, s}^{*}\right|, \hat{n}=$ $\hat{t}_{s} /\left|\hat{t}_{, s}\right|$ and $\hat{b}=\hat{t} \times \hat{n}$. Unlike curvilinear frames in the forward VC kineamtics $(R)$; for the Frenet - Serret frame in the inverse $\mathrm{VC}$ kinematics $\left(R^{*}\right)$, the frame local twist around $\hat{t}$ is a combination of a physical geometrical twist due to torsional stresses and a purely mathematical twist due to the side bending of the modules based on the mathematical definition of $\hat{b}$. To cancel the mathematical twist and to find the physical curvilinear frame $\left(\left[\hat{d}_{2}, \hat{d}_{1}, \hat{d}_{3}\right]\right)$, we use

$$
R_{\mathrm{r}}^{*}=R^{*} \cdot \Pi_{i=1}^{n_{\mathrm{p}}} R_{\phi_{i}}^{\mathrm{T}},
$$

where $\Pi$ is the post-multiplication operator, $n_{\mathrm{p}}$ is segment number, $R_{\phi}$ is a $3 \times 3$ rotation matrix around $\hat{j}, \phi=$ $\operatorname{atan}\left(\sqrt{3}\left(p_{2}+p_{3}-2 p_{1}\right) /\left(p_{2}-p_{3}\right)\right)-\pi / 2$ is the angle of the bending plane due to internal pressure in the local crosssection plane [5] and $p$ is the pressures vector in a pneumatic actuator [5] or tendon forces vector in a tendon driven design [3], [4]. UNlike [6], There is no need for a transformation between the segments. Strain rates ( $v$ and $u$ ) relationship with the loads is found from the system mechanical map.

\section{B. Static Model: Cosserat Rod and Beam Theory}

The Cosserat rod model derives the equilibrium between the forces in an infinitesimal element of a continuum media using a free body diagram as in Fig. 2 and expressed in the inertial frame as [7]

$$
\begin{aligned}
n_{, s}+f & =\sigma a_{\mathrm{c}_{\mathrm{d}}} \rho_{, t t} \\
m_{, s}+\rho_{, s} \times n+\tau & =J_{d} \omega_{, t},
\end{aligned}
$$

where $f$ and $\tau$ are the uniform distributed force and torque on the element, $\sigma$ is the material density, $a_{\mathrm{c}_{\mathrm{d}}}$ is the cross-section deformed area, $J_{\mathrm{d}}$ is the the deformed cross-section second moment of inertia and $\omega$ is the element angular velocity. The time-dependent acceleration terms $\left(\rho_{, t t}\right.$ and $\left.\omega_{, t}\right)$ are zero in a static model. Hooke's law for the linear stress-strain relation is used to relate the variation of the element boundary force $\left(n_{, s}\right)$ and moment $\left(m_{, s}\right)$ to the strain rates $([v, u])$ as

$$
n=K_{v} v, m=K_{u} u,
$$

where $K_{v}=\operatorname{diag}\left(a_{\mathrm{c}}[G, E, G]\right)$ and $K_{u}=$ $\operatorname{diag}\left(\left[E J_{\hat{d}_{2}}, G J_{\hat{d}_{1}}, E J_{\hat{d}_{3}}\right]\right)$ are diagonal stiffness matrices in the physical curvilinear frame, and the module active cross-section area $\left(a_{\mathrm{c}}\right)$ and second moment of inertias $\left(J_{\hat{d}_{1}}, J_{\hat{d}_{2}}, J_{\hat{d}_{3}}\right)$ are

$$
\begin{aligned}
a_{\mathrm{c}} & =\pi\left(r_{\mathrm{c}_{2}}^{2}-r_{\mathrm{c}_{1}}^{2}-6 r_{\mathrm{p}_{1}}^{4}\right), \\
J_{o} & =\pi / 4\left(r_{\mathrm{c}_{2}}^{4}-r_{\mathrm{c}_{1}}^{4}-6 r_{\mathrm{p}_{1}}^{2}\right), J_{\hat{d}_{1}}=2 J_{o}-6 a_{\mathrm{p}} r_{o}^{2}, \\
J_{\hat{d}_{2} \mid \hat{d}_{3}} & =J_{o}-2 a_{\mathrm{p}} r_{J \hat{d}_{2} \mid \hat{d}_{3}}^{\mathrm{T}} \cdot r_{J \hat{d}_{2} \mid \hat{d}_{3}},
\end{aligned}
$$

where $a_{\mathrm{p}}=\pi r_{\mathrm{p}}^{2}$ is the pressure chamber cross-section area, $r_{J \hat{d}_{2} \mid \hat{d}_{3}}=r_{o}\left[\mathrm{~S}\left|\mathrm{C}_{(0)}, \mathrm{S}\right| \mathrm{C}_{(2 \pi / 3)}, \mathrm{S} \mid \mathrm{C}_{(-2 \pi / 3)}\right]^{\mathrm{T}}$ and $\mathrm{S} \mid \mathrm{C}_{(x)}=$ $\sin \mid \cos (x)$. Substituting Eq. (4) in (5) and rearranging to find $v_{, s}$ and $u_{, s}$ as a function of the loads, results in a static mechanical map [7] for which a simple version for the planar case is presented in [6].

To integrate the BVP formed by combining the static and kinematics map in (Eq. (1-5)) [7] over time and space, the initial boundary values for the system strain rates and spatial configuration $(u, v, \rho, R)$ is needed. Initial values for $\rho$ and $R$ are known at the manipulator base $(s=0)$, while the strain rates $(u$ and $v)$ are known at the tip $(s=l)$. A numerical solver can be used to find initial boundary values in the equilibrium point at one of manipulator ends. Alternatively, a discrete finite element approaches can be used to solve a 
large algebraic equation for the states of all the elements at once. Duriez presented a real-time approach for this method using the sparse format of the coefficient matrices[20]. Both methods are not suitable for control and design purposes with high computational cost.

Beam theory method is a simpler approach based on the resultant stresses due to the loads at each element which results in the same equations as the Cosserat rod method, but for $v$ and $u$, unlike $v_{, s}$ and $u_{, s}$ in Eq. (4) and (5).

$$
\begin{aligned}
& v=K_{v_{\mathrm{d}}}{ }^{-1} \cdot\left(R_{\mathrm{r}}^{* \mathrm{~T}} \cdot\left(f_{\mathrm{b}}+f_{L}\right)+f_{p}\right) \\
& u=K_{u_{\mathrm{d}}}{ }^{-1} \cdot\left(R_{\mathrm{r}}^{* \mathrm{~T}} \cdot\left(\tau_{\mathrm{b}}+\tau_{L}\right)+\tau_{p}\right),
\end{aligned}
$$

where $f_{L}$ is the external load exerting at $s=s_{L}, s_{L}>s$, $f_{\mathrm{b}}=\sigma a_{\mathrm{c}}(l-s) \mathrm{g} \hat{j}$ is the body weight load, $f_{p}=p a_{\mathrm{p}} \cdot[0,1,0]^{\mathrm{T}}$ is the pneumatic pressure force, $\tau_{\mathrm{L}}=\left(\rho_{\left(s_{L}\right)}-\rho_{(s)}\right) \times f_{\mathrm{L}}$ is the moment of $f_{\mathrm{L}}, \tau_{\mathrm{b}}=\sigma a_{\mathrm{c}} \int_{s}^{l}\left(\rho_{(s)}-\rho_{(\varepsilon)}\right) \mathrm{d} \varepsilon \times \mathrm{g} \hat{j}$ is the body weight moment, $\tau_{p}=p a_{\mathrm{p}} \cdot\left[r_{J \hat{d}_{2}}, 0, r_{J \hat{d}_{3}}\right]_{\mathrm{d}}$ is the pneumatic pressure moment and $\lambda_{\hat{d}_{1}, s}=v_{\hat{d}_{1}}+1 . v_{\hat{d}_{1}}$ from the previous integration step is used in $\lambda_{\hat{d}_{1}, s}$ to prevent added nonlinearity to the equations. The downside of the beam theory approach is that we need to guess the equilibrium geometry to find the cross-sections' load, compared to the Cosserat rod model where only the initial boundary values are needed. However, it is suitable for the forward spatial and time integration using an approximate series-solution $\left(\rho^{*}\right)$. Note that $R_{\mathrm{r}}^{*}$ is used in Eq. 7 for the mechanical map; and $R$ is used in Eq. 1-2 to solve the forward kinematic map. In the equilibrium state, the geometry found from the forward kinematic map $(\rho)$ should mach the guessed geometry $\left(\rho^{*}\right)$, used for the inverse kinematic in the mechanical map $\left(\rho \approx \rho^{*}\right)$.

\section{Dynamic Model: Principle of Virtual Work}

Among all possible changes in the states of a system, the system follows the one set that minimizes the system action $(w)$. This is known as the Principle of Virtual Work or the Principle of Least Action. The spacial integration over the backbone from the summation of all the virtual works in one element of the continuum manipulator maintains an equilibrium

$$
0=\int_{s}\left(\sum_{i} \mathrm{~d} w_{i, q}\right),
$$

where $\mathrm{d} w_{i, q}$ is the differential virtual work of the $i^{\text {th }}$ action in each element derived using the corresponding load $(f)$ and virtual displacement $\left(x_{, q}\right)$ vector as $\mathrm{d} w_{q}=f \cdot x_{, q}^{\mathrm{T}}$.

The gas pressure virtual work $\left(\mathrm{d} w_{p, q}=C_{p, q} p \mathrm{~d} s\right.$, where $\left.C_{p}=C_{p_{v}, q}+C_{p_{u}, q}\right)$ is the summation of the axial virtual work with

$$
C_{p_{v}, q}=a_{p}[1,1,1]^{\mathrm{T}} v_{\hat{d}_{1}, q},
$$

and the bending virtual work is

$$
C_{p_{u}, q}=a_{p}\left(r_{J \hat{d}_{2 \mathrm{~d}}} u_{\hat{d}_{2}, q}+r_{J \hat{d}_{3 \mathrm{~d}}} u_{\hat{d}_{3}, q}\right) .
$$

The body shell virtual work $\left(\mathrm{d} w_{\mathrm{c}, q}=\mathrm{d} w_{\mathrm{c}_{v}, q}+\mathrm{d} w_{\mathrm{c}_{u}, q}\right)$, including the actuators' chamber body, is the summation of the axial virtual work

$$
\mathrm{d} w_{\mathrm{c}_{v}, q}=E_{\mathrm{d}} a_{\mathrm{c}_{\mathrm{d}}} v_{\hat{d}_{1}} v_{\hat{d}_{1}, q} \mathrm{~d} s,
$$

using the Euler-Bernoulli linear strain-stress assumption or

$$
\mathrm{d} w_{\mathrm{c}_{v}, q}=(E / 6) a_{\mathrm{c}_{\mathrm{d}}}\left(\left(v_{\hat{d}_{1}}+1\right)^{2}+2 /\left(v_{\hat{d}_{1}}+1\right)-3\right){ }_{, q} \mathrm{~d} s,
$$

based on the Neo-Hookean method assuming a symmetric uniaxial deformation rates [14], [22], and the bending virtual work

$$
\mathrm{d} w_{\mathrm{c}_{u}, q}=K_{u_{\mathrm{d}}} u u_{, q} \mathrm{~d} s,
$$

using the Euler-Bernoulli assumption.

The virtual work of gravitational body load is

$$
\mathrm{d} w_{\mathrm{b}, q}=\sigma a_{\mathrm{c}} \mathrm{g} y, q \mathrm{~d} s .
$$

The virtual work due to an external force $\left(\mathrm{d} w_{L, q}=\right.$ $\left.\mathrm{C}_{f_{L}, q} f_{L} \mathrm{~d} s\right)$ and torque $\left(\mathrm{d} w_{\tau_{L}, q}=\mathrm{C}_{\tau_{L}, q} \tau_{\mathrm{e}} \mathrm{d} s\right)$ expressed in the inertial frame and exerting at $s=s_{L}$ with $0 \leq s \leq s_{L}$ are

$$
\mathrm{C}_{f_{L}, q}=\rho_{, s q}, \quad \mathrm{C}_{\tau_{L}, q}=u_{, q} .
$$

A vector form for the body inertia virtual work can be found using the TMT method [24]. Here, the position vectors in the Cartesian space $(\rho)$ are mapped to the state space $(q)$ by $T=\left[\rho_{, q} \bar{\omega}\right]^{\mathrm{T}}$, where $\bar{\omega}$ is found by rearranging $\omega$ as $\omega=$ $\bar{\omega} q_{, t}$. The mass matrix for disk elements with an infinitesimal thickness is $\left.\mathrm{d} M=\operatorname{diag}\left(\left[\begin{array}{lll}1 & 1 & 1\end{array}\right] a_{\mathrm{c}} J_{\mathrm{d}}\right]\right) \sigma \mathrm{d} s$. Following the TMT derivations in [24], the transformed mass matrix is

$$
\mathrm{d} M_{q}=T^{\mathrm{T}} \mathrm{d} M T \mathrm{~d} s,
$$

and the transformed inertial damping matrix is

$$
\mathrm{d} D_{m, q}=T^{\mathrm{T}} \mathrm{d} M\left(T q_{, t}\right)_{, q} \mathrm{~d} s .
$$

Then the inertial damping term is $\mathrm{d} d_{m, q}=\mathrm{d} D_{m, q} q_{, t}$, and the total inertial virtual work is $\mathrm{d} w_{m}=\left(\mathrm{d} M_{q} q_{, t t}+\mathrm{d} d_{m, q}\right) \mathrm{d} s$.

The linear and angular viscous damping of the material is $\mathrm{d} w_{\mu, q}=C_{\mu} q, t \mathrm{~d} s$, where

$$
C_{\mu}=\left[\begin{array}{ll}
v & u
\end{array}\right]_{, q}^{\mathrm{T}} \operatorname{diag}\left(\left[\mu_{v} \mu_{u}\right]\right)\left[\begin{array}{ll}
v & u
\end{array}\right]_{, q},
$$

where $\mu_{v}$ and $\mu_{u}$ are the material linear and rotational viscous damping coefficient.

The vector form of the dynamic EOM in each element is

$$
\begin{array}{r}
\overbrace{\mathrm{d} M_{q} q, t t}^{\text {Acceleration }}+\overbrace{\mathrm{d} d_{m, q}+\mathrm{d} w_{\mu, q}}^{\text {Viscous Damping }}+\overbrace{\mathrm{d} w_{\mathrm{c}, q}-\mathrm{d} w_{\mathrm{g}, q}}^{\text {Conservative Actions }} \\
=\underbrace{\mathrm{d} w_{p, q}+\mathrm{d} w_{f_{L}, q}+\mathrm{d} w_{\tau_{L}, q}}_{\text {Non-conservative Actions }}
\end{array},
$$

which is integrated along the backbone curve at each time step. We use a trapezoidal rule for the spatial and a $4^{\text {th }}$ order Runge-Kutta method for the time numerical integration.

\section{Ritz AND RitZ-Galerkin Methods}

Direct single shooting, multiple shooting and concatenation methods are used to find weak-form solutions for a BVP, in which the separation of variables method is used to separates the time and space domains and makes the forward integration possible. Using the direct single shooting method, an approximate series-solution is assumed for the backbone kinematics $\left(\rho^{*}\right)$ with a finite number of terms that satisfies the initial and boundary conditions

$$
\rho^{*}=\sum_{i} c_{(t) i} \psi_{(s) i},
$$


where $c_{(t) i}$ is a time dependent coefficient which is constant in the static case and $\psi_{(s) i}$ is a spatial shape function.

Based on the Ritz method for BVPs, widely being used in the finite element analysis [25], Eq. 20 is substituted in the BVP equation, Eq. 7 for the static or Eq. 19 for dynamic model, and the coefficients $\left(c_{(t) i}\right)$ that approximate the BVP with minimum error are found. Numerical nonlinear solvers, i.e. Matlab "fsolve" function, can be used to find the coefficients $\left(c_{i}\right)$ that minimize the approximation error in the static case. The coefficients' time series $\left(c_{(t) i}\right)$ are found from the time numerical integration of the EOM in the dynamic case.

A better approximation is possible with the Continuous Galerkin method of weighted residuals, known as the RitzGalerkin method, where the weighted residuals of the system are minimized instead of the system function itself. The shape functions $\left(\psi_{(s) i}\right)$ are considered as the weighting functions $\left(W_{i}=\psi_{(s) i}\right)$ for the Ritz-Galerkin method [25]. The weighted version of Eq. (1) for the static case is

$$
0=\int_{0}^{l} W \rho_{, s}^{*} \mathrm{~d} s-\int_{0}^{l} W R\left(v+[0,1,0]^{\mathrm{T}}\right) \mathrm{d} s,
$$

where $W$ is the weighting matrix and each coordinate equation is weighted with the weighting functions of the same coordinate in the series-solution. In the dynamic case, both sides of Eq. (19) is multiplied by (W.T) to transfer the EOM from the generalized state space $(q)$ to the Cartesian state space and weight the equation for each coordinate with the corresponding weighting functions.

Tunay used the Galerkin series-solution in a discrete finite element domain for numerical simulation of the static deformation and inflation of continuum manipulators in the presence of conservative forces. He achieve a two order of magnitude improve in the computation efficiency compared to a common large deformation finite element model. However, the choice of a 7-D linear shape function and the discrete finite element approach make his method hard to be interpreted physically and unsuitable for inverse mechanics, path planning, control and stability analysis.

Based on experimental observations, we present a continuous and easy to interpret series solution where the coefficients of a series of Lagrange polynomial shape functions are assumed to be the system states $\left(q=c_{(t)}\right)$ for optimization in the static case or time integration in the dynamic case. A Lagrange polynomial $\left(\rho^{*}\right)$ is fit to three points, at the manipulator base $\left(\rho_{(0, t)}=\left[x_{0}, y_{0}, z_{0}\right]=[0,0,0]\right)$, middle $\left(\rho_{(l / 2, t)}=\right.$ $\left.\left[x_{1(t)}, y_{1(t)}, z_{1(t)}\right]\right)$ and at the tip $\left(\rho_{(l, t)}=\left[x_{2(t)}, y_{2(t)}, z_{2(t)}\right]\right)$ of the form

$$
\begin{aligned}
\rho^{*} & =\sum_{j=0}^{2}\left(\psi_{j(s)} \cdot\left[x_{j(t)}, y_{j(t)}, z_{j(t)}\right]^{\mathrm{T}}\right), \\
\psi_{j(s)} & =C_{\psi} \cdot \prod_{m}\left(\frac{s-s_{m}}{s_{j}-s_{m}}\right), 0 \leq m \leq N, m \neq j .
\end{aligned}
$$

where $C_{\psi}=\operatorname{diag}\left(\left[s / s_{j}, 1,1\right]\right)$ is a modification coefficient guaranteeing the manipulator axis to be perpendicular at the base $\left(\hat{t}_{0}=\hat{j}\right)$, the points' position vector $\left(q=c_{(t)}=\right.$ $\left.\left[x_{1(t)}, y_{1(t)}, z_{1(t)}, x_{2(t)}, y_{2(t)}, z_{2(t)}\right]\right)$ is the constant coefficients in the static model or the time dependent coefficients as the system generalized state vector for the dynamic model, $\psi_{j(s)}$ is the shape function and the weighting matrix for the RitzGalerkin method is $W=\left[\psi_{1(s)}, \psi_{2(s)}\right]^{\mathrm{T}}$.

\section{NONLINEAR CONTROL AND OBSERVATION}

A unified vector form for the modeling, nonlinear control and observer design, based on feedback linearization method is derived. Defining the input pressure and external load vector as the system states and adding algebraic constraints for the desired controls to the system EOM, for Eq. (19) after the spatial integration, we have

$$
\begin{gathered}
{\left[\begin{array}{ccc}
M_{q} & -\left[\begin{array}{ccc}
C_{p} & C_{f_{L}} & C_{\tau_{L}}
\end{array}\right] \\
A_{1} & A_{2}
\end{array}\right]\left[\begin{array}{c}
q_{, t t} \\
q_{p} \\
q_{f_{L}} \\
q_{\tau_{L}}
\end{array}\right]=} \\
\\
{\left[\begin{array}{c}
-d_{m, q}-w_{\mathrm{s}, q}+w_{\mathrm{g}, q}-w_{\mu, q} \\
B
\end{array}\right],}
\end{gathered}
$$

where $A_{1}$ and $A_{2}$ are matrices resulting from algebraic relations for the constraint or desired controls, $B$ is the desired and/or input value vector, $q_{p}, q_{f_{L}}$ and $q_{\tau_{L}}$ are the input pressure, external force and torque vector, introduced as system states. The coefficient matrix is $\left[3 n_{q}+9\right] \times\left[3 n_{q}+9\right]$, $A_{1}$ is a $9 \times 3 n_{q}$ and $A_{2}$ is a $9 \times 9$ matrix and $B$ is a $9 \times 1$ vector where $n_{q}$ is the number of states. $A$ and $B$ are adjusted according to the required modeling, control and/or observation tasks and can be switched easily to achieve complex control strategies. Eq. (23) is numerically integrated w.r.t time by having the initial values for $\left[q, q_{p}, q_{f_{\mathrm{e}}}, q_{\tau_{\mathrm{e}}}\right]$. The control or observation values for $\left[q_{p}, q_{f_{\mathrm{e}}}, q_{\tau_{\mathrm{e}}}\right]$ is found by two times numerical differentiation of the integration results w.r.t time, since they are integrated twice alongside the system EOM.

For simple forward simulation we have $A_{1}=\mathbf{0}, A_{2}=I$ and $B=\left[\begin{array}{lll}p & f_{L} & \tau_{L}\end{array}\right]^{\mathrm{T}}$, where $I$ is the identity matrix. In the case of tip position control in the presence of external loads, we have $A_{1}\left(3 n_{q}+1, n_{q}\right)=1, A_{1}\left(3 n_{q}+2,2 n_{q}\right)=1, A_{1}\left(3 n_{q}+\right.$ $\left.3,3 n_{q}\right)=1, A_{2}\left(3 n_{q}+4:\right.$ end $\left., 4: 9\right)=I$ and $B=\left[\left(\rho_{\mathrm{tip}_{\mathrm{h}}, t t}+\right.\right.$ pid) $\left.f_{L} \tau_{L}\right]^{\mathrm{T}}$, where $\rho_{\mathrm{tip}_{\mathrm{h}}, t t}$ is the desired tip position and pid is a PID or any other feedback control term. Configuration control can be achieved by changing $A_{1}$ to control the position vector of any other intermediate point on the backbone.

Force control at the tip using the pressure inputs and for a known tip torque $\left(\tau_{L}\right)$ and position profile $\left(\rho_{\text {tip }, t t}\right)$ is possible by setting $A_{1}\left(3 n_{q}+1, n_{q}\right)=1, A_{1}\left(3 n_{q}+2,2 n_{q}\right)=$ $1, A_{1}\left(3 n_{q}+3,3 n_{q}\right)=1, A_{2}\left(3 n_{q}+4: e n d, 4: 9\right)=I$ for the tip vector position control and $B=\left[\rho_{\text {tip }, t t}\left(f_{L_{\mathrm{h}}}+\text { pid }\right) \tau_{L}\right]^{\mathrm{T}}$, where $f_{L_{\mathrm{h}}}$ is the desired force vector. For the tip torque control $\left(\tau_{L_{\mathrm{h}}}\right)$, we have the same $A$ matrix with $B=\left[\rho_{\mathrm{tip}, t t} f_{(} \tau_{L_{\mathrm{h}}}+\right.$ pid) $]^{\mathrm{T}}$.

Simultaneous force and position control is possible by fast enough switching between different control scenarios or having a combination of non-overlapping force and position directions to derive a proper control matrix set for.

Shape-based force estimation is possible by proper adjustment of the matrices. For example, for the tip force estimation based on the tip position vector we have $A_{1}\left(3 n_{q}+4, n_{q}\right)=$ 
TABLE I

STIFF-FLOP PARAMETERS.

\begin{tabular}{l|c|l|c|l|c} 
Sym. & Value & Sym. & value & Sym. & value \\
\hline$r_{\mathrm{p} 1}[\mathrm{~mm}]$ & 2.25 & $r_{\mathrm{p} 2}[\mathrm{~mm}]$ & 3.0 & $r_{\mathrm{o}}[\mathrm{mm}]$ & $8.5(9)^{*}$ \\
$r_{\mathrm{c} 2}[\mathrm{~mm}]$ & $12.5(12)$ & $r_{\mathrm{c} 1}[\mathrm{~mm}]$ & 4 & $l[\mathrm{~mm}]$ & 44 \\
$l_{\mathrm{fs}}[\mathrm{mm}]$ & $10(17)$ & $m[\mathrm{gram}]$ & 24.5 & $m_{\mathrm{e}}[\mathrm{gram}]$ & 11 \\
$\mathrm{~g}\left[\mathrm{~m} / \mathrm{s}^{2}\right]$ & 9.81 & $E_{\mathrm{sta}}[\mathrm{KPa}]$ & $205^{* *}$ & $\sigma\left[\mathrm{Kg} / \mathrm{m}^{3}\right]$ & 1300 \\
$\mu_{v}[\mathrm{Ns} / \mathrm{m}]$ & $1 \mathrm{e}-3^{* *}$ & $\mu_{u}[\mathrm{Ns} / \mathrm{rad}]$ & $4 \mathrm{e}-5^{* *}$ & &
\end{tabular}

* Values in brackets are for the tests with 3D deformation (Fig. 5d,e).

** Parameters found from identification to minimize the simulation errors.

$1, A_{1}\left(3 n_{q}+5,2 n_{q}\right)=1, A_{1}\left(3 n_{q}+6,3 n_{q}\right)=1, A_{2}\left(3 n_{q}+1:\right.$ $\left.3 n_{q}+3,1: 3\right)=I, A_{2}\left(3 n_{q}+7: 3 n_{q}+9,7: 9\right)=I$ and $B=\left[p\left(\rho_{\text {tip }, t t}+\text { pid }\right) \tau_{L}\right]^{\mathrm{T}}$, in which the known tip geometry is assumed as geometrical constraint and the PID term is for numerical analysis error compensation not as a term for physical feedback control.

\section{ExPerimental Results AND Discussion}

We used a STIFF-FLOP (STIFFness controllable Flexible and Learn-able manipulator for surgical OPerations) pneumatic actuator module [26] as a standard, simple yet accurate and durable design without inhomogeneous deformations, i.e. due to structure swallow. Similar design is used in recent research [5], [6], [17]. The measured and identified structural parameters are presented in Table I with an experimental setup similar to the one in [14], [15] (Fig. 1). We choose STIFF-FLOP modules because of their high repeatability and negligible performance change due to aging and fatigue during the experimental trials. However, accurate modeling of these modules are challenging due to inhomogeneities as a result of the fabrication process.

Experimental conditions and inputs are used in the simulations where Matlab "fsolve", "ode113" and "fmincon" are used for direct single shooting optimization, time integration and parameter identification. 33 static points and 100 [s] dynamic motion recorded data of a module in planar motion with extensive external load (Fig. 1 and 3) and 43 static points and 200 [s] dynamic motion recorded data of a multi-segment manipulator in general 3D motion with one active module (Fig. 4.a-d and 5) are used to verify the accuracy of our model. Seven points from the static test are used to identify $E$ and $\mu_{v \mid u}$. Error is defined as the distance between the tip position in the model and experiments divided by the initial length of the manipulator as a reference length [6]. High external load brings the manipulator to an "S" shape configuration in the planar motion case (Fig. 1). Using a polynomial of order three, an average $6 \%(\approx 4[\mathrm{~mm}])$ mean error is observed for the static model in $2 \mathrm{D}$ and $8 \%(\approx 6[\mathrm{~mm}])$ in $3 \mathrm{D}$ motion using both the Ritz and Ritz-Galerkin method. For the Cosserat beam method, Our method shows to be at least twist faster and as accurate as a numerical interpolation solution with the same number of nodes [9]. Despite Tunay's conclusion, a Galerkin solution that works fine in the static case cannot easily be extended to a dynamic problem [21] and we found that the Ritz-Galerkin solution in the dynamic case is not stable and results in large errors, but the Ritz solution maintains a mean error of $20 \%(\approx 14[\mathrm{~mm}])$ in the $2 \mathrm{D}$ and $16 \%(\approx 12$ [mm]) in the 3D case throughout the dynamic test. The mean accuracy of the Ritz and Ritz-Galerkin solutions are similar in the static case with no significant different in the simulation speed while the Ritz solution in dynamic case is more accurate than for the static case, $14 \%$ in the planar and $8 \%$ in the 3D motion. This difference is due to large inertial effect in the dynamic case which shows the need and importance of a more accurate damping model to be considered in our future work. The models are more accurate for lower input pressures. Inaccuracy in the fabrication of the STIFF-FLOP module causes that the experimental results show out of plane deformation in the $z$ direction in the planar motion and twist in the general 3D motion, despite a symmetric actuation strategy and planar external force. This deformation is not captured in our model since any term to model the parameters' inaccuracy is not considered. Considering the effect of the manipulator axial highly elastic deformation on the segments shear and elasticity modulus results in a $6 \%$ increase in the model accuracy; however, a higher value for the elasticity modulus is identified compared to our previous works [14], [15], [9]. Our modeling results in the planar case shows to be the most accurate solution compared to CC (31\% error), PVW using CC kinematics (28\%), the Cosserat rod model (6-12\%) and the approximate solutions similar to [8] $(11 \%)$ in the static case and compared to the lumped system model (22\% error) in the dynamic case [9]. The series-solution with two points (four states in a planar motion and six states in a general 3D motion) shows to be efficient and accurate enough to predict the "S" shape configuration of the manipulator under significant external loads. However, simulations for general 3D motion of a multi-segment manipulator with two active modules (Fig. 4.e) shows the different results from polynomial of order four and five (Fig. 6). This shows the importance of a proper choice for the polynomial order, especially, where the manipulator needs to perform in the confined maze-like space of a minimally invasive surgery as the final goal of our research. Using Matlab software on a regular laptop computer, the direct single shooting algorithm usually takes about 2 [s] to find the equilibrium configuration for a single-curve formation and 3 [s] for a double-curve formation ("S" shape) in the static case, and almost the same time as the actual experiment duration for the dynamic case. Compared to the presented established methods in the literature, our method has the highest static and a high dynamic modeling accuracy with exceptional real-time computational performance which shows the feasibility of real-time implementation of this approach in real-world applications. Our weak-from solution provides a powerful analytical means for sensitivity analysis of the model performance w.r.t. the system parameters for structural design optimization. We observed a slight change in the module cross-section dimensions affect the accuracy by $2 \%$ similar to [15] while a change in the module stiffness module, i.e. not considering the elasticity reduction due to axial highly elastic deformation, affect the accuracy by up to $6 \%$. A detailed sensitivity analysis, as in [15], will be carried out in the future of our research to predict how inaccuracy in the model parameters can affect the simulation results for a successful control system design. This model is derived from a framework 

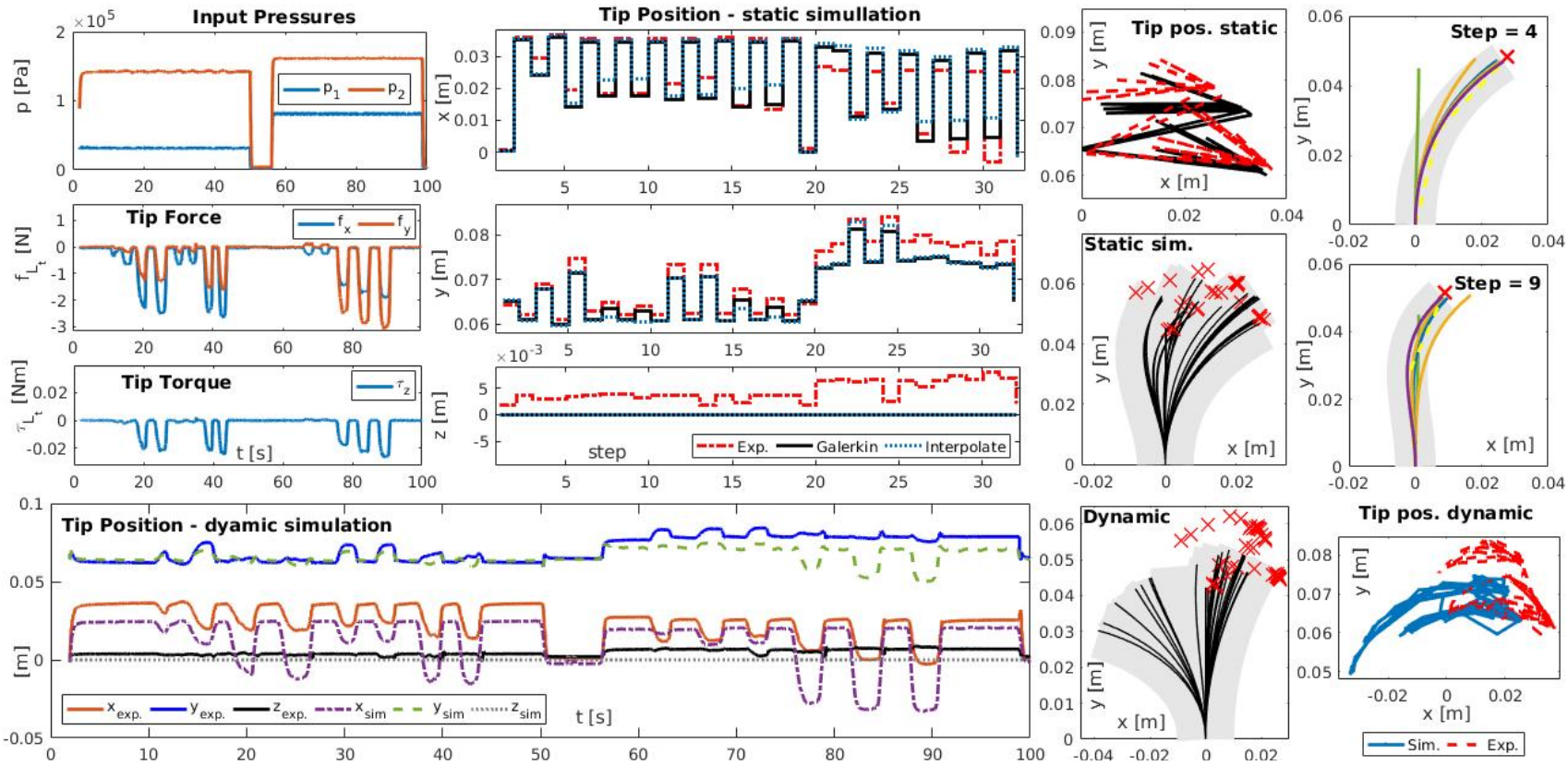

Fig. 3. Results for planar deformation of a module with extensive external load at the tip in static and dynamic case (Fig. 1): pressure inputs, external force and torque at the tip, tip position time series and in the task space, simulation frames, and single shooting iterations for steps 4 and 9 in the static case.

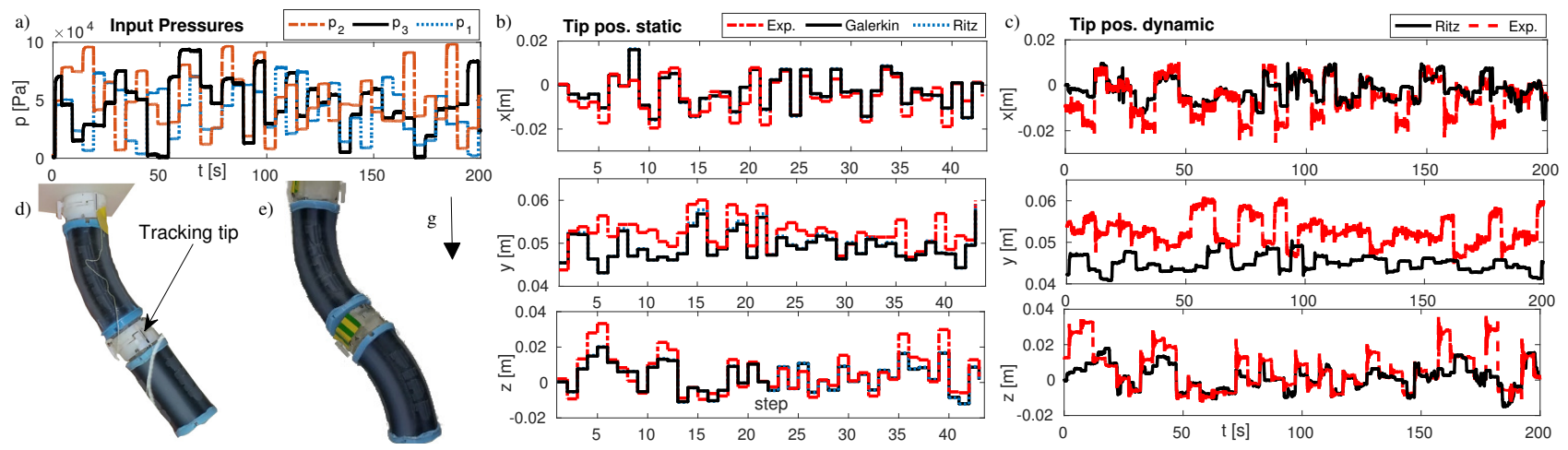

Fig. 4. Results for general 3D deformation of a multi-segment manipulator in static and dynamic case: pressure inputs (a) and tip position time series in static (b) and dynamic (c) case for the manipulator with one actuated module (d), manipulator with two actuated modules (e).
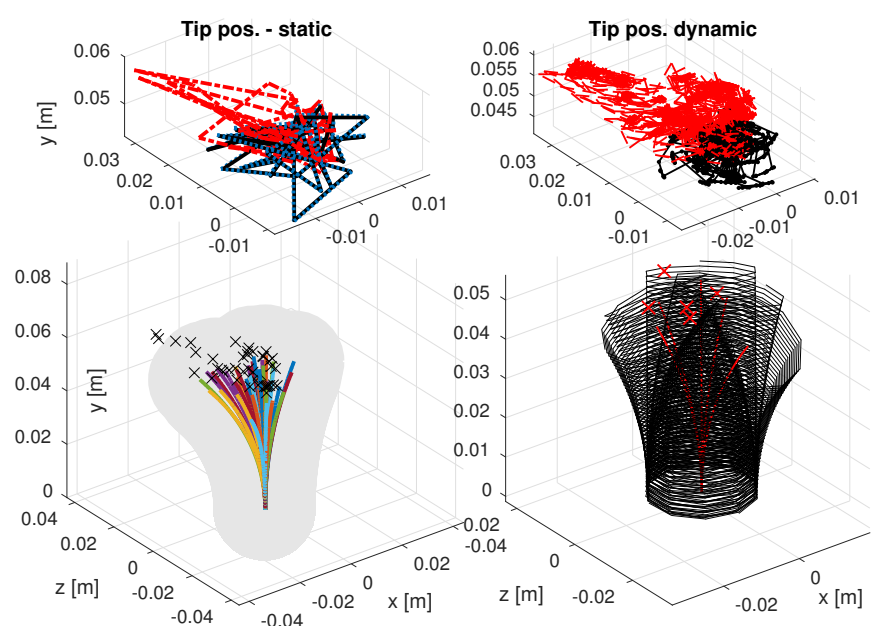

Fig. 5. Tip position in the task space and simulation frames for general 3D motion of multi-segment manipulator with one active module (Fig. 4.d) in static (left) and dynamic (right) case.

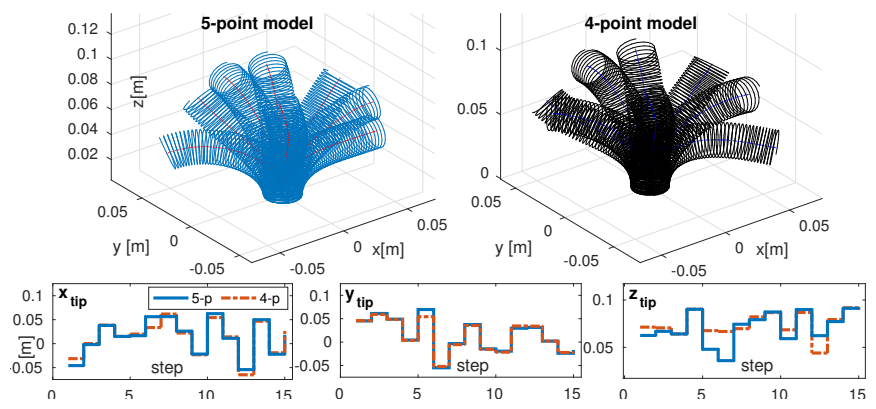

Fig. 6. Tip position time series and simulation frames for a multi-segment manipulator with two active modules in general 3D motion (Fig. 4.e) in static case with a modified Lagrange polynomial of order four (4-p: left) and five (5-p:right).

with coaxial homogeneity in the manipulator cross-section and any other anomaly due to fabrication, aging and fatigue with a stochastic nature will be compensated with a feedback control term. These affects can be addressed using statistical approach 
and by incorporating stochastic terms in the future control model. A precise forward model contributes in increasing the stability, reducing the controller gains, better estimation of the system states and compensation of measurement noises. After using the absolute error for the verification and comparison of our model in the first step, we are going to investigate the performance of our model (Eq. 23) for force estimation and control with application in soft tissue palpation and stiffness imaging. A continuum manipulator with 5-15 [gram-force] and 6-43\% estimation error, achievable with STIFF-FLOP modules [27], is showed to be successful in such tasks [28]. The controller repeatability and durability are important for such tasks which we plan to address in our future work.

\section{CONCLUSION}

In this paper, a modified Lagrange polynomial seriessolution, based on experimental observation, was presented to produce a new technique for deriving the Cosserat rod static and Lagrangian dynamic model of a continuum manipulator. Our approach featured a small number of states (six in our analysis) which are the Cartesian positions of two points on the manipulator (at the tip and in the middle). We used a direct single shooting method to solve the Cosserat rod static model and forward numerical integration for the Lagrange dynamic model using the Ritz and Ritz-Galerkin approach. A unified vector form for the manipulator Lagrange dynamics was derived using which the modeling, control and observation scenarios can be easily implemented by adjusting three control matrices and a vector for the desired and input values. Noting the importance of considering the manipulator axial highly elastic deformation, the Ritz method is found to be more accurate in dynamic cases while the Ritz-Galerkin method is slightly more accurate in the static cases. The model is compared with experimental results and five other well-known models and shown to be the most accurate and efficient approach with the smallest possible number of states, suitable for real-time static and dynamic modeling and controller design. We plan to use this method to control a continuum manipulator for biological tissue palpation and stiffness imaging in medical applications.

\section{REFERENCES}

[1] S. Hirose and M. Mori, "Biologically inspired snake-like robots," in Robotics and Biomimetics, ROBIO 2004. IEEE International Conference on, (Shenyang, China), pp. 1-7, IEEE, 2004.

[2] D. Rus and M. T. Tolley, "Design, fabrication and control of soft robots," Nature, vol. 521, no. 7553, pp. 467-475, 2015.

[3] F. Maghooa, A. Stilli, Y. Noh, K. Althoefer, and H. A. Wurdemann, "Tendon and pressure actuation for a bio-inspired manipulator based on an antagonistic principle," in 2015 IEEE International Conference on Robotics and Automation (ICRA), (Seattle, WA, USA), pp. 2556-2561, IEEE, May 2015.

[4] A. Ataollahi, R. Karim, A. S. Fallah, K. Rhode, R. Razavi, L. D. Seneviratne, T. Schaeffter, and K. Althoefer, "Three-Degree-of-Freedom MR-Compatible Multisegment Cardiac Catheter Steering Mechanism," IEEE Transactions on Biomedical Engineering, vol. 63, pp. 2425-2435, Nov. 2016.

[5] R. J. Webster and B. A. Jones, "Design and Kinematic Modeling of Constant Curvature Continuum Robots: A Review," The International Journal of Robotics Research, vol. 29, no. 13, pp. 1661-1683, 2010.

[6] D. Trivedi, A. Lotfi, and C. D. Rahn, "Geometrically Exact Models for Soft Robotic Manipulators," IEEE Transactions on Robotics, vol. 24, pp. 773-780, Aug. 2008.
[7] M. Neumann and J. Burgner-Kahrs, "Considerations for Follow-theLeader Motion of Extensible Tendon-driven Continuum Robots," 2016.

[8] I. S. Godage, D. T. Branson, E. Guglielmino, G. A. Medrano-Cerda, and D. G. Caldwell, "Shape function-based kinematics and dynamics for variable length continuum robotic arms," in 2011 IEEE International Conference on Robotics and Automation (ICRA), (Shanghai, China), pp. 452-457, IEEE, May 2011.

[9] S. Sadati, S. E. Naghibi, A. Shiva, I. D. Walker, K. Althoefer, and T. Nanayakkara, "Mechanics of Continuum Manipulators, A Comparative Study of Five Methods with Experiments," (Surrey, UK), Springer, 2017.

[10] I. S. Godage, R. Wirz, I. D. Walker, and R. J. Webster, "Accurate and Efficient Dynamics for Variable-Length Continuum Arms: A Center of Gravity Approach," Soft Robotics, vol. 2, pp. 96-106, Sept. 2015.

[11] Y. Shapiro, A. Wolf, and K. Gabor, "Bi-bellows: Pneumatic bending actuator," Sensors and Actuators A: Physical, vol. 167, pp. 484-494, June 2011.

[12] J. Fra, J. Czarnowski, M. Macia, and J. Glwka, "Static modeling of multisection soft continuum manipulator for stiff-flop project," in Recent Advances in Automation, Robotics and Measuring Techniques, vol. 267, pp. 365-375, Springer, 2014

[13] E. Tatlicioglu, I. D. Walker, and D. M. Dawson, "Dynamic modelling for planar extensible continuum robot manipulators," in IEEE International Conference on Robotics and Automation (ICRA), (Rome, Italy), pp. 1357-1362, IEEE, 2007.

[14] S. Sadati, A. Shiva, A. Ataka, S. E. Naghibi, I. D. Walker, K. Althoefer, and T. Nanayakkara, "A Geometry Deformation Model for Compound Continuum Manipulators with External Loading," in 2016 IEEE International Conference on Robotics and Automation (ICRA), (Stockholm, Sweden), pp. 4957-4962, IEEE, 2016.

[15] S. Sadati, S. E. Naghibi, A. Shiva, Y. Noh, A. Gupta, I. D. Walker, K. Althoefer, and T. Nanayakkara, "A Geometry Deformation Model for Braided Continuum Manipulators," Frontiers in Robotics and AI, vol. 4, June 2017

[16] J. Burgner-Kahrs, D. C. Rucker, and H. Choset, "Continuum Robots for Medical Applications: A Survey," IEEE Transactions on Robotics, vol. 31, pp. 1261-1280, Dec. 2015.

[17] I. S. Godage, G. A. Medrano-Cerda, D. T. Branson, E. Guglielmino, and D. G. Caldwell, "Dynamics for variable length multisection continuum arms," The International Journal of Robotics Research, vol. 35, no. 6, pp. 695-722, 2016.

[18] G. Chen, M. T. Pham, and T. Redarce, "Sensor-based guidance control of a continuum robot for a semi-autonomous colonoscopy," Robotics and Autonomous Systems, vol. 57, no. 6-7, pp. 712-722, 2009.

[19] I. S. Godage, E. Guglielmino, D. T. Branson, G. A. Medrano-Cerda, and D. G. Caldwell, "Novel modal approach for kinematics of multisection continuum arms," in IEEE International Conference on Intelligent Robots and Systems (IROS), (San Francisco, California, USA), pp. 10931098, IEEE, 2011. 00034.

[20] C. Duriez and T. Bieze, "Soft Robot Modeling, Simulation and Control in Real-Time," in Soft Robotics: Trends, Applications and Challenges, vol. 12 of Biosystems \& Biorobotics, pp. 103-109, Springer, 1 ed., 2017.

[21] I. Tunay, "Spatial continuum models of rods undergoing large deformation and inflation," IEEE Transactions on Robotics, vol. 29, no. 2, pp. 297-307, 2013. 00000 .

[22] A. N. Gent, Engineering with rubber: how to design rubber components. Munich, Germany: Hanser Publishers, 2012.

[23] P. Polygerinos, Z. Wang, J. T. B. Overvelde, K. C. Galloway, R. J. Wood, K. Bertoldi, and C. J. Walsh, "Modeling of Soft Fiber-Reinforced Bending Actuators," IEEE Transactions on Robotics, vol. 31, pp. 778789, June 2015.

[24] S. Sadati, S. Naghibi, and M. Naraghi, "An Automatic Algorithm to Derive Linear Vector Form of Lagrangian Equation of Motion with Collision and Constraint," Procedia Computer Science, vol. 76, pp. 217 222, 2015 .

[25] J. S. Hesthaven, S. Gottlieb, and D. Gottlieb, Spectral methods for timedependent problems, vol. 21. Cambridge University Press, 2007.

[26] J. Fra, J. Czarnowski, M. Macia, J. Gwka, M. Cianchetti, and A. Menciassi, "New STIFF-FLOP module construction idea for improved actuation and sensing," in 2015 IEEE International Conference on Robotics and Automation (ICRA), pp. 2901-2906, May 2015.

[27] T. Ranzani, G. Gerboni, M. Cianchetti, and A. Menciassi, "A bioinspired soft manipulator for minimally invasive surgery," Bioinspiration \& Biomimetics, vol. 10, p. 035008, May 2015.

[28] A. Bajo and N. Simaan, "Hybrid motion/force control of multi-backbone continuum robots," The International Journal of Robotics Research, p. 0278364915584806 , July 2015. 